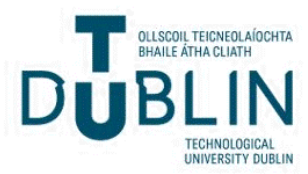

Technological University Dublin

ARROW@TU Dublin

2004-01-01

\section{A Simple Phase-Shifting Lateral Shearing Interferometer}

\author{
Emilia Mihaylova \\ Technological University Dublin, emilia.mihaylova@tudublin.ie \\ Maurice Whelan \\ Institute for Health and Consumer Protection, European Commission \\ Vincent Toal \\ Technological University of Dublin, vincent.toal@tudublin.ie
}

Follow this and additional works at: https://arrow.tudublin.ie/cieoart

Part of the Engineering Commons

\section{Recommended Citation}

Mihaylova, E., Whelan, M. \& Toal, V. (2004) A Simple Phase-Shifting Lateral Shearing Interferometer. Optics Letters, vol. 29 no.11, pp. 1264. doi:10.1364/OL.29.001264

This Article is brought to you for free and open access by the Centre for Industrial and Engineering Optics at ARROW@TU Dublin. It has been accepted for inclusion in Articles by an authorized administrator of ARROW@TU Dublin. For more information, please contact arrow.admin@tudublin.ie, aisling.coyne@tudublin.ie, gerard.connolly@tudublin.ie.

Funder: Technological Sector Research Programme Strand III supported by the Irish Government

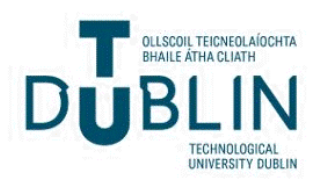




\title{
A SIMPLE PHASE-SHIFTING LATERAL SHEARING INTERFEROMETER
}

\author{
Emilia Mihaylova
}

Centre for Industrial and Engineering Optics, Dublin Institute of Technology,Kevin Street, Dublin 8, Ireland; e-mail: emilia.mihaylova@dit.ie

Maurice Whelan

Institute for Health and Consumer Protection, European Commission, DG - Joint Research Centre, 21020 Ispra, Italy; e-mail: maurice.whelan@jrc.it

Vincent Toal

Centre for Industrial and Engineering Optics, Dublin Institute of Technology,Kevin Street, Dublin 8, Ireland; e-mail: vincent.toal@dit.ie

\begin{abstract}
A phase-shifting electronic speckle pattern shearing interferometer with a very simple shearing device is proposed. Two partially reflective glass plates are used to introduce the shear in this new interferometer. The reflection coefficients of the coatings were 0.3 and 0.7 respectively. The distance between the two glass plates controls the size of the shear. The proposed new interferometric system is simple, flexible and low cost.
\end{abstract}

OCIS codes: 120.6160 Speckle interferometry; 220.4830 Optical systems design

Electronic speckle pattern shearing interferometry (ESPSI) enables direct measurements of displacement derivatives to be made with variable sensitivity ${ }^{1-2}$. A common lateral shear interferometer uses a plane parallel glass plate as a shearing device. The amount of shear is selected by the thickness and tilt of the plate ${ }^{3}$. The disadvantages of this simple shearing interferometer are that the resulting interferogram has low fringe visibility and phase-shifting techniques can not be applied. Phase-shifting shearography is more sensitive than the simple 
digital shearography ${ }^{4}$ and can provide three-dimensional (3D) plots of the displacement derivatives ${ }^{1}$.

When two light waves interfere, the following equation ${ }^{5}$ relates their relative phase $\Phi$ at a location to their relative geometrical path length $L$ :

$$
\Phi=\frac{2 \pi}{\lambda} n L-\beta
$$

where $\lambda$ is the wavelength of the laser light, $n$ is the refractive index of the medium through which the laser light is transmitted, and $\beta$ is a constant phase. The change in the relative phase $\Delta=\delta \Phi$ or phase change, which manifests as visible fringes, can be effected by an incremental change in any of the three parameters $\lambda, n$, and $L$. Thus,

$$
\Delta=\frac{\partial \Phi}{\partial \lambda} \delta \lambda+\frac{\partial \Phi}{\partial n} \delta n+\frac{\partial \Phi}{\partial L} \delta L=-\frac{2 \pi L n}{\lambda^{2}} \delta \lambda+\frac{2 \pi L}{\lambda} \delta n+\frac{2 \pi n}{\lambda} \delta L
$$

where $\delta \lambda$, $\delta$, and $\delta L$ denote respectively, the incremental change in wavelength, in refractive index, and in relative geometrical path length of the interfering waves.

If the same wavelength is used and the test environment is still air $(n=1)$, only the $\delta L$ term in Eq. (2) is nonzero, resulting in the following equation for the phase change ${ }^{6}$ :

$$
\Delta=\frac{2 \pi}{\lambda}[A \delta u+B \delta v+C \delta w]
$$

where $u, v$ and $w$ are the displacement components of the neighboring point $P^{\prime}(x+\Delta x, y, z+\Delta z)$ relative to point $P(x, y, z)$ on the test surface, and $A, B$, and $C$ are sensitivity factors that are related to the optical arrangement. For small image shearing $\Delta x$, the displacement terms in Eq. (3) can be expressed in terms of partial derivatives:

$$
\Delta=\frac{2 \pi}{\lambda}\left[A \frac{\partial u}{\partial x}+B \frac{\partial v}{\partial x}+C \frac{\partial w}{\partial x}\right]
$$


In our case (Fig. 1) the illuminating object beam lies in the (x, z) plane, so there is no sensitivity to displacement along y axis so long as the beam is well collimated.

The phase change is:

$$
\Delta=\frac{2 \pi}{\lambda}\left[A \frac{\partial u}{\partial x}+C \frac{\partial w}{\partial x}\right]
$$

Digital phase shifting can be implemented to provide fringe analysis. There are a number of algorithms available for the purpose. The algorithm we use is a five bucket type in which four interferograms are captured having phase shifts of $\pi / 2, \pi, 3 \pi / 2$ between successive ones. A fifth interferogram with zero phase shift is captured after the loading is applied and the phase map corresponding to Eq.5 is obtained from these data ${ }^{7}$.

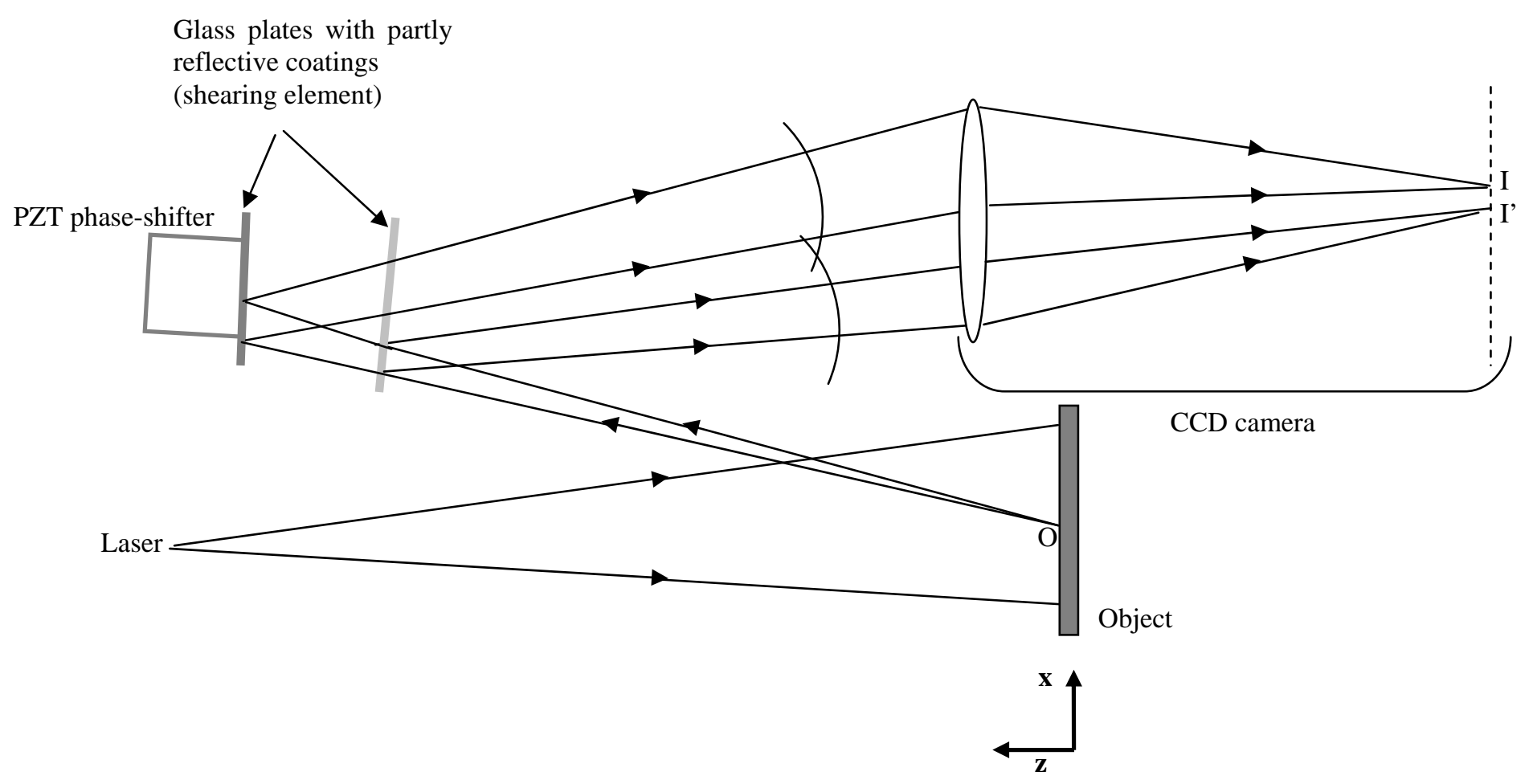

Fig. 1. Schematic diagram of the ESPSI system with two partially reflective glass plates as a shearing element: I and I' are the laterally sheared images of $\mathrm{O}$ on the CCD faceplate. 
The arrangement of the proposed phase-shifting ESPSI system with two partially reflective glass plates is presented in Figure 1. A CW Nd-YVO 4 laser $(\lambda=532 \mathrm{~nm})$ is used as the light source. A laser beam illuminates the object at an angle to the normal to the object surface so that the system is sensitive to displacement in both $\mathrm{x}$ and $\mathrm{z}$ directions. The reflection coefficients of the coatings are 0.3 for the first glass plate and 0.7 for the second glass plate. In this way the two sheared images have approximately the same intensity and the fringe contrast is very good. The second glass plate is mounted on a piezoelectric transducer (PZT). This phase-shifter allows the introduction of a known phase shift. The consecutive speckle shearing interference patterns are recorded by a CCD camera and stored in a computer. The phase difference (after subtraction) is displayed as a phase map. The phase-shifting ESPSI system is controlled remotely by software ${ }^{8}$.

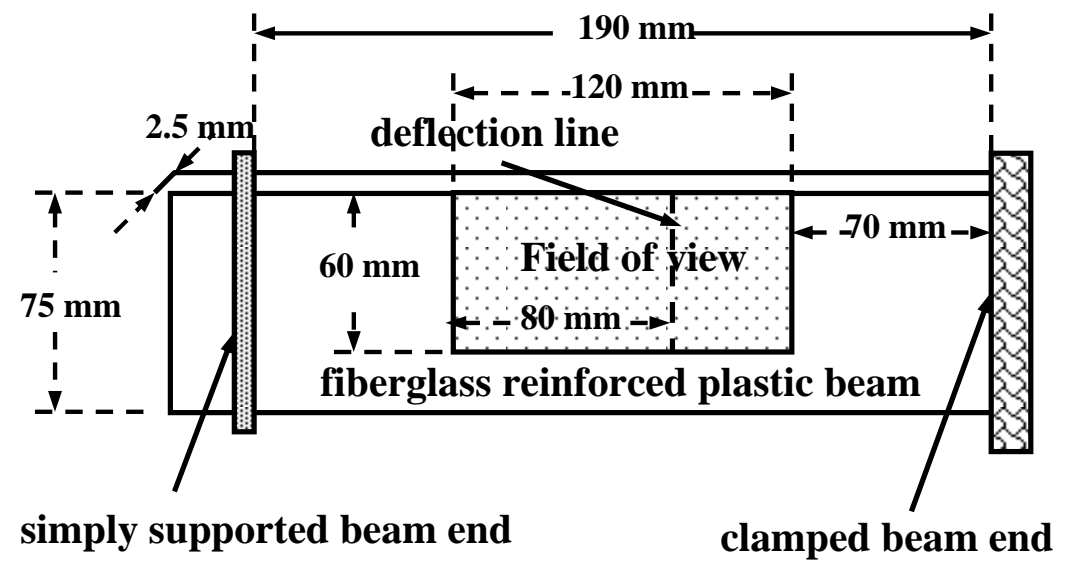

Fig. 2. A scheme of the FRP sample.

We used a bending test of a fibreglass reinforced plastic (FRP) beam to demonstrate the phaseshifting ESPSI system with two glass plates. One end of the beam is clamped, the other end is simply supported (Fig. 2). It is intended to carry out a rigorous analysis of the beam behaviour in three and four point bending. The field of view is $120 \mathrm{~mm} \mathrm{x} 60 \mathrm{~mm}$. The shear is $\Delta x=8 \mathrm{~mm}$. 
Figure 3 presents results obtained from the ESPSI system. The fringes are observed in real time and can be analyzed as in ESPI by recording a series of phase-shifted speckle-sheared fringe patterns. The obtained fringe patterns are good quality and show consistency with the increase of the deflection. The phase at each point is evaluated ${ }^{7}$ from the phase data. The $3 \mathrm{D}$ plot of the phase distribution is obtained from these data. The phase map is indicative of both out of plane displacement gradient and in plane displacement gradient because the illuminating beam is at an angle to the normal.

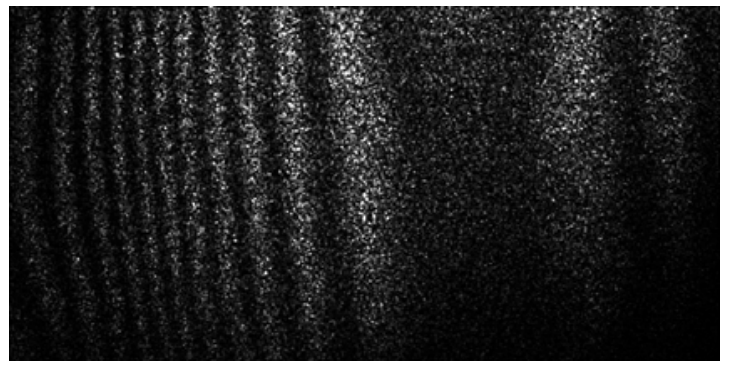

a

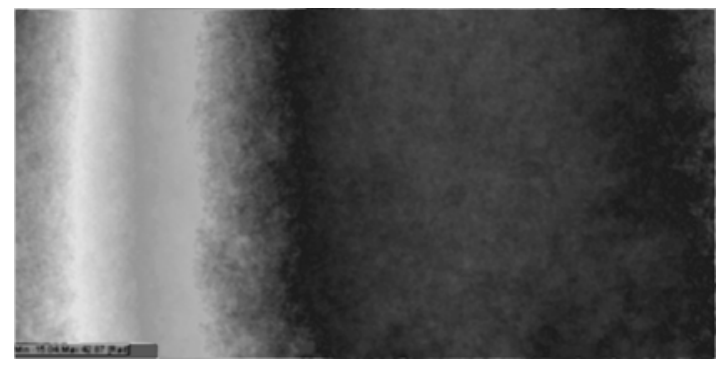

C

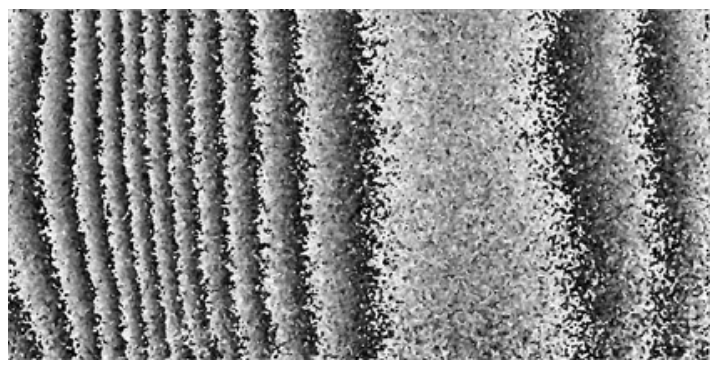

b

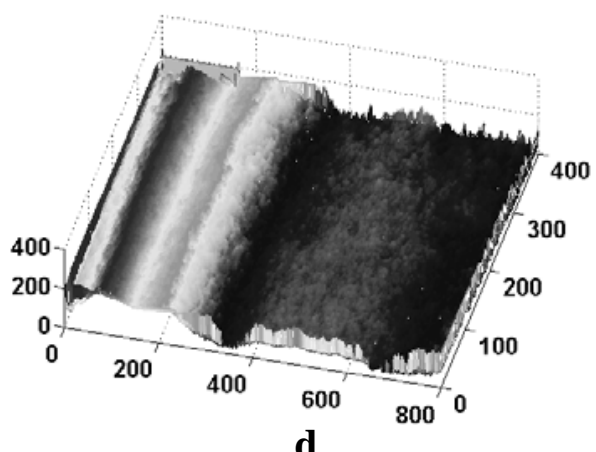

Fig. 3. ESPSI fringes on FRP beam during bending, recorded with ESPSI system with two glass plates under deflection of $20 \mu \mathrm{m}$ : a) ESPSI fringes; b) wrapped phase map; c) unwrapped phase map; d) psedo-3D plot of the phase distribution. 
The ESPSI system using glass plates to shear the image has some advantages compared to the commercially available systems based on a combination of a beam splitter and two mirrors (modified Michelson interferometer). It is simple and flexible and offers a simple way to introduce discrete shear steps between two images. An additional advantage is the low cost of such a system. The ESPSI system with two partially reflective glass plates is compact and suitable for industrial applications. It is flexible and easy to adjust to examine small or large object areas. The results obtained with this simple phase-shifting interferometer are promising for future applications to engineering metrology.

Acknowledgements are made to Technological Sector Research Programme Strand III supported by the Irish Government. Emilia Mihaylova would like to thank the Arnold F. Graves Scholar Programme at Dublin Institute of Technology.

\section{REFERENCES}

1. W. Steinchen and L. Yang, "Digital shearography: theory and application of digital speckle pattern shearing interferometry”, SPIE, Washington (2003).

2. R. S. Sirohi, "Speckle methods in experimental mechanics”, in Speckle Metrology, Ed. R. S. Sirohi, Mercel Dekker, New York (1993).

3. J. C. Wyant, “A simple interferometric MFT instrument”, Optics Comm. 19 (1), 120-121 (1976).

4. M. Owner-Petersen, "Decorrelation and fringe visibility: on the limiting behaviour of various electronic speckle pattern correlation interferometers”, J. Opt. Soc. Am. 8, 1082-1089 (1991).

5. C. M. Vest, Holographic Interferometry, John Wiley, New York (1979).

6. Y. Y. Hung and C. Y. Liang, "Image shearing camera for direct measurement of surfacestrains”, Appl. Opt. 10 (7), 1046-1050 (1979).

7. P. K. Rastogi, “Digital Speckle Pattern Interferometry and Related Techniques”, Ed. P. K. Rastogi, John Wiley \& Sons Ltd., New York (2001).

8. EspiTest Software, developed by Andreas Langhoff (http://www.daedalussoft.com) and Maurice Whelan, Copyright (c) 1998 European Commission, DG - Joint Research Centre, Ispra, Italy. 